\title{
Fungal Metabolite, Epoxyquinol B, Crosslinks Proteins by Epoxy-thiol Conjugation
}

\author{
Hiroshi Kamiyama, Takeo Usui, Masakazu Uramoto, Hiroshi Takagi, Mitsuru Shoji ${ }^{\dagger}$, \\ Yujiro Hayashi, Hideaki Kakeya ${ }^{\dagger \dagger}$, Hiroyuki Osada
}

Received: January 7, 2008 / Accepted: February 8, 2008

(C) Japan Antibiotics Research Association

\begin{abstract}
Epoxyquinol B (EPQB) is a fungal metabolite, containing two $\alpha, \beta$-epoxy ketones. We previously showed that EPQB inhibited the signal transduction involved in angiogenesis through the binding to cysteine residues of receptor kinases. However, the inhibitory mechanism was undefined. In this report, we found that one EPQB molecule is covalently bound to two $L$-cysteine molecules through two epoxide residues on EPQB. Furthermore, EPQB crosslinked binding proteins through the cysteine residues. These results suggest that EPQB inhibits receptor kinases by crosslinking with other protein or by intramolecular crosslinking.
\end{abstract}

Keywords epoxyquinol B, crosslink, epoxide, cysteine, epoxyquinoid, fungal metabolite

It is well known that epoxides can be opened by direct attack of the nucleophile on the epoxide ring. Epoxide containing compounds are able to directly form stable adducts with proteins containing thiol or phenolic group, under mild experimental conditions [1]. We previously showed that epoxyquinol B (EPQB), a fungal metabolite and the epoxyquinoid containing two $\alpha, \beta$-epoxy ketones, inhibited angiogenesis through the covalent binding to cysteine residues of VEGFR2, EGFR, FGFR, and PDGFR $\beta$ [2 4]. In this paper, we report the reaction mechanism between EPQB and cysteine residues of binding proteins, resulted in crosslinking of the binding proteins.

EPQB possesses the highly reactive functional group; two $\alpha, \beta$-epoxy ketones which could form covalent bonds with nucleophiles such as a thiol (Fig. 1A). Therefore, we anticipated that EPQB has several reaction sites with cysteine residue of the binding protein. To investigate this possibility, we analyzed the reaction products of EPQB and $L$-cysteine by LC/MS analysis. EPQB and $L$-cysteine were incubated together in water for 10, 30, 60, and 120 minutes. Then, the products were analyzed by QTRAP LC/MS/MS system. As shown in Fig. 1B, four major peaks were detected in a time dependent manner. $L$-cysteine and EPQB were detected with $\mathrm{m} / \mathrm{z} 120$ and $387,\left([\mathrm{M}-\mathrm{H}]^{-}\right)$with retention times (Rt) of 1.74 and 29.2 minutes, respectively. Two $L$-cysteine adducts of EPQB were detected, and
H. Osada (Corresponding author), H. Kamiyama, T. Usui, M. Uramoto, H. Takagi, H. Kakeya: Antibiotics Laboratory, RIKEN Discovery Research Institute, 2-1 Hirosawa, Wako, Saitama 351-0198, Japan, E-mail: hisyo@riken.jp

H. Kamiyama, H. Osada: Graduate School of Science and Engineering, Saitama University, 255 Shimo-okubo, Sakura-ku, Saitama 338-8570, Japan

T. Usui: Graduate School of Life and Environmental Sciences, University of Tsukuba, 1-1-1 Tennodai, Tsukuba, Ibaraki 3058572, Japan
M. Shoji, Y. Hayashi: Department of Industrial Chemistry, Faculty of Engineering, Tokyo University of Science, Kagurazaka, Shinjuku-ku, Tokyo 162-8601, Japan

$\dagger$ Present address: Department of Chemistry, Graduate School of Science, Tohoku University, 6-3 Aramaki-Aza, Aoba, Sendai 9808578, Japan

${ }^{\dagger}$ Present address: Department of System Chemotherapy and Molecular Sciences, Division of Bioinformatics and Chemical Genomics, Graduate School of Pharmaceutical Sciences, Kyoto University, 46-29 Yoshida-Shimoadachi, Sakyo-ku, Kyoto 6068501, Japan 
A

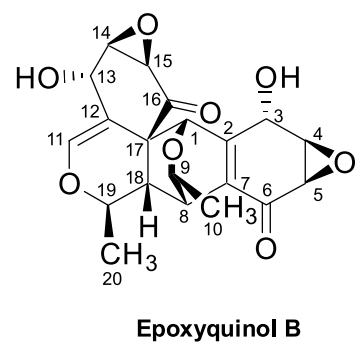

B

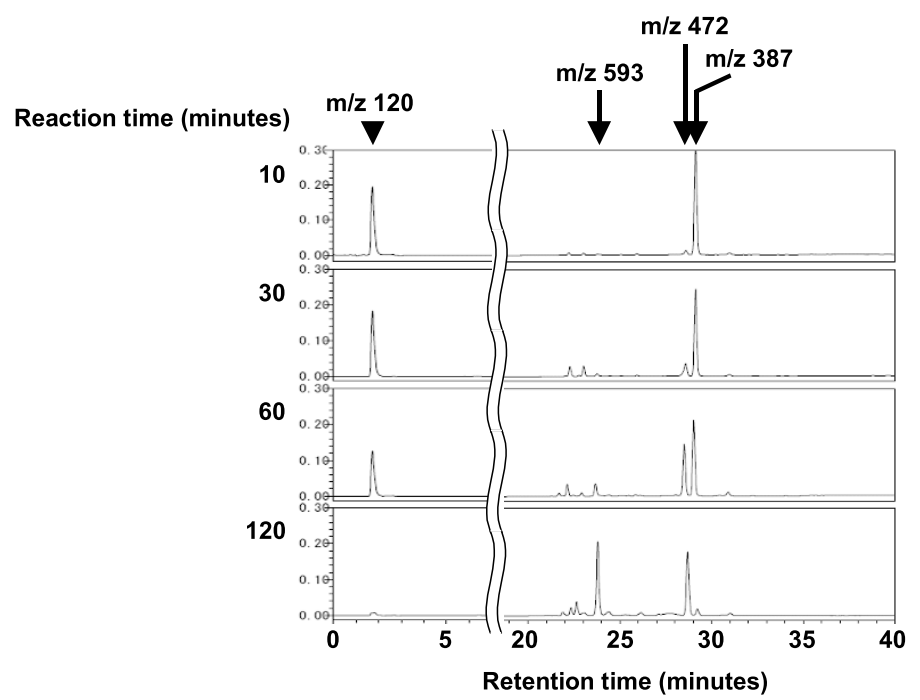

Fig. 1 HPLC and LC/MS analysis of reaction products between EPOB and L-cysteine.

(A) Structure of Epoxyquinol B (EPQB). (B) HPLC and LC/MS analyses of reaction of EPQB and cysteine. $10 \mathrm{mM} E P Q B$ and $100 \mathrm{mM}$ L-cysteine were incubated in water for $10,30,60$, and 120 minutes at $4^{\circ} \mathrm{C}$. The samples were separated by XTerra MSC18 column. HPLC and LC/MS systems were carried out by Allians 2695 Separation Module (Waters) and Q TRAP LC/MS/MS System (Applied Biosystems) with XTerra MSC18 column by using a gradients of $0.05 \%$ formic acid and acetonitrile (2 100\%).

determined as $\left[\mathrm{EPQB}+2 \mathrm{Cys}-2 \mathrm{H}_{2} \mathrm{O}-\mathrm{H}\right]^{-}$(Rt: 23.8 minutes, $\mathrm{m} / \mathrm{z}:$ 593.0) and [EPQB $\left.+1 \mathrm{Cys}-2 \mathrm{H}_{2} \mathrm{O}-\mathrm{H}\right]^{-}$(Rt: 28.7 minutes, $m / z: 472.0$ ), respectively. These results suggest that one EPQB molecule is sequentially bound to two cysteine molecules through two of its reactive sites. The UV spectra of EPQB, EPQB $+1 \mathrm{Cys}-2 \mathrm{H}_{2} \mathrm{O}$, and $\mathrm{EPQB}+2 \mathrm{Cys}-2 \mathrm{H}_{2} \mathrm{O}$ show absorption maxima at 237,225 , and $220 \mathrm{~nm}$, respectively (data not shown), indicating that these compounds containing the same chromophore derived from a $\alpha, \beta$-unsaturated ketone. Therefore, it was strongly suggested that this conjugated system retained in the molecules of two reaction products and thus the reaction sites on EPQB are two epoxides. To confirm this possibility, we analyzed the structures of two reaction products. EPQB was incubated with $L$-cysteine for 1 and 4 hours, by ${ }^{1} \mathrm{H}$ NMR. Since it was difficult to isolate enough amount of these products for NMR analysis, the reaction mixture was lyophilized, dissolved again in methanol- $d_{4}$ and prepared for the measurement of ${ }^{1} \mathrm{H}$ and DQF-COSY spectra by a Jeol JNM ECP-500 NMR spectrometer. The ${ }^{1} \mathrm{H}-\mathrm{NMR}$ data revealed that the ratio of integration value between methyl doublets of EPQB $(1.24 \mathrm{ppm}, J=6.4 \mathrm{~Hz})$, and each product (product 1: $1.26 \mathrm{ppm}, J=6.4 \mathrm{~Hz}$, and product 2: $1.28 \mathrm{ppm}$, $J=6.4 \mathrm{~Hz}$ ) was two to one. As the ${ }^{1} \mathrm{H}$ signal derived from $11-\mathrm{H}(6.4 \mathrm{ppm}, \mathrm{s})$ was still observed in the ${ }^{1} \mathrm{H}-\mathrm{NMR}$ spectra of both products, the addition site of cysteine molecule was supposed to be on two epoxide part of EPQB. (The numbering of both products was temporarily assigned on the basis of the corresponding carbon atoms of EPQB.) We focused on the analysis of the ${ }^{1} \mathrm{H}$ chemical shifts of epoxide parts in both reaction products. The assignments of $13-\mathrm{H}$, $14-\mathrm{H}$ and $15-\mathrm{H}$ were difficult because of overlapping with 
the background signals from the reaction mixture. On the other hand, the signals of $3-\mathrm{H}, 4-\mathrm{H}$ and $5-\mathrm{H}$ of products were observed separately. The signal of 4-H of EPQB $(3.80 \mathrm{ppm}, \mathrm{dd}, J=1.4,3.7 \mathrm{~Hz})$ shifted to high magnetic field (product 1: $3.63 \mathrm{ppm}$, dd, $J=1.8,3.2 \mathrm{~Hz}$, product 2: $3.62 \mathrm{ppm}$, dd, $J=1.8,2.8 \mathrm{~Hz}$ ), because of the formation of the $\mathrm{C}-\mathrm{S}$ bond in adducts instead of the $\mathrm{C}-\mathrm{O}$ bond in EPQB. The cross peak was due to the protons attached to the carbon atoms originated from $\mathrm{C} 4$ and $\mathrm{C} 5$ of EPQB. It was clearly observed in the DQF-COSY spectrum. Furthermore, the stereo-structure of the products was investigated by the Dreiding model. It was considered that the six membered ring of the products has $3-\alpha-\mathrm{OH}, 4-\beta-\mathrm{H}, 5-\beta-\mathrm{OH}$ and $\alpha, \beta-$ unsaturated ketone system, because of the subsequent ring opening of epoxide accompanied with the inversion of 4- $\mathrm{H}$. The dihedral angle of $50 \sim 55^{\circ}$ between $3-\mathrm{H}$ and $4-\mathrm{H}$ and $110 \sim 115^{\circ}$ between $4-\mathrm{H}$ and $5-\mathrm{H}$ were correlated to $J$ values as given in the NMR spectrum by the Karplus's correlation between dihedral angle and $J$ values [5]. These results suggested that the initial binding site of $L$-cysteine in EPQB was the C-4 position. The analyses of LC/MS, $\mathrm{UV}$, and NMR data indicated that one molecule of EPQB binds to two molecules of $L$-cysteine through its opening of the two epoxides.

When EPQB was treated with recombinant VEGFR2 kinase protein, we noticed that several ladder-like hypershifted bands appeared with EPQB-dependent manner (Fig. 2A). Since it was previously shown that one EPQB covalently binds to two cysteines, we anticipated that EPQB crosslinked the binding proteins through the cysteine residues. To investigate this possibility, we used cysteine/serine-mutated Vh1 related phosphatase (VHR) proteins $[6,7]$ as a model protein. VHR protein contains only four cysteine residues; Cys22, Cys30, Cys124, and Cys171. Therefore, we made two mutant VHR proteins, 3S and $4 \mathrm{~S}$; in these mutant proteins, three of the cysteines $(\mathrm{C} 22 / 30 / 124)$ and all of the cysteines $(\mathrm{C} 22 / 30 / 124 / 171)$ on VHR were replaced with serines, respectively. Wild type (WT) or each cysteine/serine mutant VHR (3S and 4S) were incubated with $10 \mathrm{mM}$ EPQB in water and VHR buffer (50 mM Tris-HCl (pH 7.5) and 1.0 mM EDTA) for 1 hour. The samples were separated with SDS-PAGE and the VHR proteins were detected by western blotting analysis. As shown in Fig. 2B, ladder-like hypershifted bands were observed in EPQB-treated WT VHR proteins. On the contrary, only dimerized band of VHR was appeared when EPQB was treated with $3 \mathrm{~S}$ mutant VHR protein which contains only one cysteine. Furthermore, no additional band was observed when EPQB was treated with $4 \mathrm{~S}$ mutant VHR protein. The data shown in Fig. $2 \mathrm{~B}$ was obtained in the water condition to correlate with the LC/MS
A

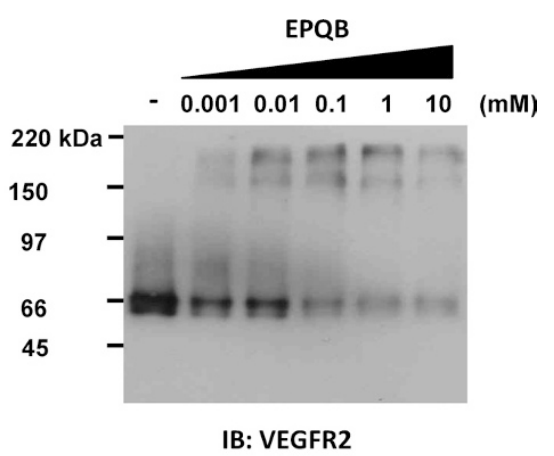

B

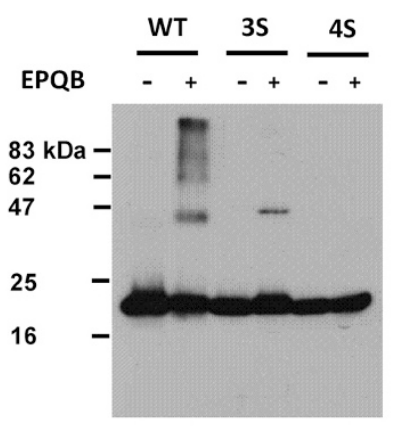

IB: VHR

Fig. 2 EPQB crosslinks VHR protein through the binding with cysteine residues.

(A) The appearance of a ladder-like hypershifted bands containing recombinant VEGFR2 kinase protein by EPQB treatment. Recombinant VEGFR2 kinase protein $(66 \mathrm{kDa})$ was treated with $0.001,0.01,0.1,1$, and $10 \mathrm{mM}$ EPQB for 2 hours in vitro. These samples were analyzed by Western blotting. (B) Crosslinking assay of EPQB. Wild type (WT) or cysteine/serine mutant (3S and 4S) VHR (400 ng) were incubated with $10 \mathrm{mM}$ EPQB in water for 1 hour. The proteins were separated with SDSPAGE and VHR proteins $(21 \mathrm{kDa})$ were detected by Western blotting.

experiment condition. In fact, there were no differences in the crosslinking pattern between the water and the VHR buffer conditions (data not shown). These results strongly suggest that EPQB crosslinked proteins through the cysteine residues on its target protein.

Recently, there have been a few reports on smallmolecule ligands containing two protein-binding surfaces. These ligands modulate a number of cellular processes especially signal transduction pathways. FK1012A, which is a dimer of FK506, is developed as a pharmacological mediator. It induced dimerization of the FK506-binding protein and consequently initiated the signaling cascade [8 10]. Furthermore, Guido L. et al. suggested that helenalin, a sesquiterpene lactone, crosslinks protein 
through the cysteine residues of NF- $\kappa \mathrm{B}$ [11]. Since the active site of NF- $\kappa \mathrm{B}$ contains cysteine residues, the binding and crosslinking by helenalin may affect the signaling pathway. Our results showed that one EPQB also binds to two cysteine residues in its binding protein(s), and suggests that EPQB may consequently inhibit angiogenesis signal transductions through inter/intramolecular crosslinking of the target proteins.

Acknowledgment This study was supported in part by Junior Research Associate, Grants for Basic Research (Chemical Biology Project) from RIKEN, and a Grant-in-Aid from the Ministry of Education, Culture, Sports, Science, and Technology of Japan. We thank Naoki Kanoh (Tohoku University) for technical advice and useful discussion.

\section{References}

1. Mateo C, Abian O, Fernández-Lorente G, Pedroche J, Fernández-Lafuente R, Guisan JM, Tam A, Daminati M. Epoxy sepabeads: a novel epoxy support for stabilization of industrial enzymes via very intense multipoint covalent attachment. Biotechnol Prog 18: 629-634 (2002)

2. Kakeya H, Onose R, Yoshida A, Koshino H, Osada H. Epoxyquinol B, a fungal metabolite with a potent antiangiogenic activity. J Antibiot 55: 829-831 (2002)

3. Kakeya H, Onose R, Koshino H, Yoshida A, Kobayashi K, Kageyama S, Osada H. Epoxyquinol A, a highly functionalized pentaketide dimer with antiangiogenic activity isolated from fungal metabolites. J Am Chem Soc 124: 3496-3497 (2004)

4. Kamiyama H, Kakeya H, Usui T, Nishikawa K, Shoji M, Hayashi Y, Osada H. Epoxyquinol B shows antiangiogenic and antitumor effects by inhibiting VEGFR2, EGFR, FGFR, and PDGFR. Oncology Res (in press)

5. Karplus M. Contact electron-spin coupling of nuclear magnetic moments. J Chem Phys 30: 11-15 (1959)

6. Ishibashi T, Bottaro DP, Chan A, Miki T, Aaronson SA. Expression cloning of a human dual-specificity phosphatase. Proc Natl Acad Sci USA 89: 12170-12174 (1992)

7. Ueda K, Usui T, Nakayama H, Ueki M, Takio K, Ubukata M, Osada H. 4-Isoavenaciolide covalently binds and inhibits VHR, a dual-specificity phosphatase. FEBS Lett 525: 48-52 (2002)

8. Spencer DM, Wandless TJ, Schreiber SL, Crabtree GR. Controlling signal transduction with synthetic ligands. Science 262: 1019-1024 (1993)

9. Diver ST, Schreiber SL. Single-step synthesis of cellpermeable protein dimerizers that activate signal transduction and gene expression. J Am Chem Soc 119: 5106-5109 (1997)

10. Blau CA, Peterson KR, Drachman JG, Spencer D. A proliferation switch for genetically modified cells. Proc Natl Acad Sci USA 94: 3076-3081 (1997)

11. Guido L, Knorre A, Schmidt TJ, Pahl HL, Merfort I. The anti-inflammatory sesquiterpene lactone helenalin inhibits the transcription factor NF- $\kappa \mathrm{B}$ by directly targeting $\mathrm{p} 65$. J Biol Chem 273: 33508-33516 (1998) 